\title{
Dynamics of Propagation Phenomena in Biological Pattern Formation
}

\author{
G. Liţcanu ${ }^{a, b 1}$ and J. J. L. Velázquez ${ }^{c}$ \\ ${ }^{a}$ Institute of Mathematics "O. Mayer”, Romanian Academy, 700505 Iaşi, Romania \\ ${ }^{b}$ Institute of Applied Mathematics and Mechanics \\ Warsaw University, 02-097 Warsaw, Poland \\ ${ }^{c}$ Departamento de Matemática Aplicada, Facultad de Matemáticas \\ Universidad Complutense, 28040 Madrid, Spain
}

\begin{abstract}
A large variety of complex spatio-temporal patterns emerge from the processes occurring in biological systems, one of them being the result of propagating phenomena (see, for example [35]). This wave-like structures can be modelled via reaction-diffusion equations. If a solution of a reaction-diffusion equation represents a travelling wave, the shape of the solution will be the same at all time and the speed of propagation of this shape will be a constant. Travelling wave solutions of reaction-diffusion systems have been extensively studied by several authors from experimental, numerical and analytical points-of-view (see, for example, [14], [16], [18], [22], [23], [24], [41], [45], [48], [49]).

In this paper we focus on two reaction-diffusion models for the dynamics of the travelling waves appearing during the process of the cells aggregation. Using singular perturbation methods to study the structure of solutions, we can derive analytic formulae (like for the wave speed, for example) in terms of the different biochemical constants that appear in the models. The goal is to point out if the models can describe in quantitative manner the experimental observations.
\end{abstract}

Key words: reaction-diffusion systems, travelling waves, singular perturbation methods AMS subject classification: 35B25, 35K57, 92C37

\footnotetext{
${ }^{1}$ Corresponding author. E-mail: lorelai@mimuw.edu.pl
} 


\section{Introduction}

\subsection{Biological background}

One of the most important mechanisms of cell biology is the chemotaxis, the ability of the cells to migrate towards regions of higher concentrations of a chemical attractant. The cell migration is an important factor for cellular communication. Communication by chemical signals determines how cells organize themselves and has an important role in embryogenesis, development of nervous system, immunity, angiogenesis, inflammatory diseases or metastasis. The competition between the diffusive dispersal of the chemical attractant and the chemotactic aggregation can give rise to complex and varied geometric patterns.

In a vast number of natural phenomena a key element is the propagation of some substance, or some mechanical deformation like a travelling wave. Spatio-temporal wave phenomena were observed, for example, in neuromuscular activity in heart tissue, wound healing, tumor growth, embryology, cell-cell interaction, or in the aggregation in fields of social amoebae. A particulary good biological example is provided by the species Dictyostelium discoideum (Dd hereafter) which are able to alternate between unicellular and multicellular forms. One reason for the interest in these cellular organisms was caused by the fact that the processes of morphogenesis and pattern formation occur in a similar manner as in many higher organisms. They are used as model for studying cell motility and transduction, cell-type differentiation, and developmental processes. Taking into account the experimental data for the biological processes that occur in this organism (see, for example, [3], [10], [11], [15], [32], [36], [40]), there have been different mathematical models describing the developmental cycle of $D d$ (see, for instance, [7], [13], [19], [20], [28], [29], [30], [37], [38], [42], [47]).

Social amoebae $D d$ are soil organisms which feed on bacteria and multiply by binary fission. Upon depletion of their food source, individual $D d$ cells aggregate to form, first, hemisherical aggregates called mounds that evolve later to a functional multicellular organism, the slug, and culminate in a fruiting body. This process of aggregation is controlled by a signalling mechanism based in the propagation of a chemoattractant (that is cyclic adenosine 3' 5'-monophosphate, hereafter $c A M P$ ). A schematic description of the signal transduction/relay process is the following (for more details see [2], [15], [33], [38], [39], [42]). Some randomly located individual $D d$, called pacemakers, begin to secrete in a pulsatile manner $c A M P$ which propagates outward as concentric or spiral waves that spread through the population density. This extracellular signal, detected by highly specific $c A M P$ receptors of the surrounding cells, initiates a complex genetic process determining which cells become relay competent. After the extracellular $c A M P$ binds to the receptors, the complex $c A M P$-receptor activate the adenylate cyclase via G-proteins. The resulting receptor-adenylate cyclase complex catalyzes the conversion of ATP (adenosine triphosphate) to intracellular $c A M P$, part of which is secreted into the extracellular medium. Also, binding of receptors leads to a refractory period during which the cell cannot respond to constant external $c A M P$ concentration, but respond to variations of $c A M P$ concentration.

There are many mathematical models describing different stages of $D d$ development. Some of the most widely used models for $c A M P$ propagation in early stage of aggregation are Martiel- 
Goldbeter's model [33], Monk-Othmer's model [34] and Tang-Othmer's model [39]. These models are able to account for the most relevant features of $c A M P$ signals during the cell aggregation process, namely the presence of autonomous oscillations of $c A M P$, the relay of suprathreshold $c A M P$ pulses, the existence of a refractory period in which cells are unable to answer to additional stimuli. Travelling waves also exist, for certain parameter domains, in model chemotaxis mechanisms such as proposed by Keller and Segel [27] and Keller and Odell [25], [26]. However, none of these models has a confirmed molecular background, as some of the features of the biological processes are not totally measurable. Nevertheless, this temporary lack of knowledge of these processes involved does not, we think, diminish the interest of a theoretical study of a model, what is more if the results fit with the experimental data.

In a recent paper [31], we have focused on the mathematical analysis of two continuum models for $c A M P$ relay response, based on the kinetics rate laws of the Martiel-Goldbeter model [33] and Goldbeter-Segel [17], respectively. Our goal was to study, using singular perturbation methods, these models for $c A M P$ wave propagation. We have considered the one-dimensional spatial problem and we have made the assumption that extracellular $c A M P$ is the only diffusible chemical of the systems. The model proposed by Martiel and Goldbeter [33], is based on the receptor modification for the $c A M P$ signaling system that controls the process of aggregation. As in [44], in order to deal with nonspatially homogeneous solutions, we include in the model diffusion for the extracellular $C A M P$. The main results that we have obtained are formulae for the speed of the extracellular $c A M P$-pulses, for the width of the pulses, and for the time that the cells remain in the excited state. These formulae are valid in a parameter domain that is consistent with the experimental values used in the original paper [33]. We used a similar approach to study also the so-called GoldbeterSegel model [17]. In the assumption that the $c A M P$-receptor on the cell surface is a regulatory part of adenylate cyclase, this model is less realistic from the biological point of view, but we can see a rather similar underlying mathematical structure in both problems in spite of the fact that they are based in different biochemical assumptions.

After 8-10 hours, aggregation centers of $D d$ results in the formation of a mound, a complex of up to $10^{4}-10^{5}$ cells. All cells of the mound differentiate into two cell types: pre-spore and pre-stalk. Although the mechanism of cell sorting are still under debate, some experimental data suggest that the coordinated upward movement of the cells in the later stages of aggregation can by organized by $c A M P$ waves ([8], [9], [47]). Also cell-cell adhesion and cell-substratum adhesion seem to play an important role in regulating cell movements.

This paper is organized as follows. In Section 2. we briefly describe the main results that we have obtained for the existence of the pulse-like solutions in a model for $c A M P$ signalling in early stage of cells aggregation, based on the kinetics rate laws of the Martiel-Goldbeter model [33]. The detailed proofs of the mean results that are presented in this Section can be found in [31]. In Section 3. we discuss a mathematical model proposed by Vasiev and Weijer [47] for $c A M P$ signalling in mound formation. The key focus of attention is on how the pulse speed depends on the various system biological parameters in both models. 


\subsection{Asymptotic notations}

Definition 1..1. Let $f$ and $g$ be two functions defined in a neighborhood of $x_{0}$, that do not vanish on their domain.

○ The notation

$$
f(x) \ll g(x), \quad x \rightarrow x_{0}
$$

which is read " $f(x)$ is much smaller than $g(x)$, as $x$ tends to $x_{0} "$ or " $g(x)$ is much larger than $f(x)$, as $x$ tends to $x_{0} "$, means $\lim _{x \rightarrow x_{0}}[f(x) / g(x)]=0$.

- The notation

$$
f(x) \sim g(x), \quad x \rightarrow x_{0}
$$

which is read " $f(x)$ is asymptotic to $g(x)$, as $x$ tends to $x_{0} "$, means $\lim _{x \rightarrow x_{0}}[f(x) / g(x)]=1$, or, equivalently, $f(x)-g(x) \ll g(x)$ as $x \rightarrow x_{0}$.

$\circ$ The notation

$$
f(x)=\mathcal{O}[g(x)], \quad x \rightarrow x_{0}
$$

which is read " $f(x)$ is at most of order $g(x)$, as $x$ tends to $x_{0} "$, means $|f(x) / g(x)|<M$, for some constant $M$ if $x$ is in a neighborhood of $x_{0}$.

Remark 1..2. Throughout the paper we use also the notation $f(x) \simeq g(x), x \rightarrow x_{0}$ to indicate in an informal, non-rigorous manner that the functions $f$ and $g$ have the same order of magnitude.

\section{A mathematical model for cAMP signalling in early stage of cells aggregation}

\subsection{Description of the model}

In [33], Martiel and Goldbeter proposed a model which is based on receptor modification for the $c A M P$ sinalling system in $D d$, characterized by the following set of three ordinary differential equations:

$$
\begin{aligned}
\frac{d r}{d t} & =-f_{1}(u) r+f_{2}(u)(1-r) \\
\frac{d w}{d t} & =q \sigma \phi(r, u)-\left(k_{i}+k_{t}\right) w \\
\frac{d u}{d t} & =\frac{k_{t}}{h} w-k_{e} u
\end{aligned}
$$

where $r$ is the total fraction of activated receptors, $w$ is the intracellular $c A M P$ concentration and $u$ is the extracellular $c A M P$ concentration. The functions $f_{1}(u), f_{2}(u), \phi(r, u)$ are given by: 


$$
\begin{aligned}
f_{1}(u) & =\frac{k_{1}+k_{2} u}{1+u}, \quad f_{2}(u)=\frac{k_{1} L_{1}+k_{2} L_{2} c u}{1+c u} \\
\phi(r, u) & =\frac{\alpha\left(\lambda \theta+\epsilon Y^{2}\right)}{1+\alpha \theta+\epsilon Y^{2}(1+\alpha)} ; \quad Y=\frac{r u}{1+u}
\end{aligned}
$$

The various parameters appearing in the equations (2.1)-(2.3) and in the formulae (2.4), (2.5) are some biological parameters (we refer to the original article [33] for details).

In order to study the propagation of $c A M P$ pulses for a system having as underlying kinetics the equations (2.1)-(2.3), we assumed that the extracellular $c A M P$ diffuses according to the standard Fick's law. Then we obtain, in the one-dimensional case, the following system of equations:

$$
\begin{aligned}
& \frac{\partial r}{\partial t}=-f_{1}(u) r+f_{2}(u)(1-r) \\
& \frac{\partial w}{\partial t}=q \sigma \phi(r, u)-\left(k_{i}+k_{t}\right) w \\
& \frac{\partial u}{\partial t}=\frac{k_{t}}{h} w-k_{e} u+D \frac{\partial^{2} u}{\partial y^{2}}
\end{aligned}
$$

where $D$ is the diffusion coefficient and the variables $r, w$, and $u$ depend on the space $y$ and the time $t$.

The numerical parameter values which give a good quantitative agreement between experimental data and theoretical solutions of the model (2.6)-(2.8) are indicated in Table 1. All of them have been chosen from [33], except the diffusion coefficient that has been taken from [12].

\begin{tabular}{|cr|cr|}
\hline \multicolumn{4}{|c|}{ Table 1. Numerical parameter values. } \\
\hline Parameter & $\begin{array}{l}\text { Numerical } \\
\text { value }\end{array}$ & $\begin{array}{l}\text { Dimensionless } \\
\text { parameter }\end{array}$ & $\begin{array}{l}\text { Numerical } \\
\text { value }\end{array}$ \\
\hline$k_{1}$ & $0.4 \mathrm{~min}^{-1}$ & $c$ & 100 \\
$k_{2}$ & $0.004 \mathrm{~min}^{-1}$ & $L_{2}$ & 0.1 \\
$\sigma$ & $0.57 \mathrm{~min}^{-1}$ & $L_{1}\left(=c L_{2}\right)$ & 10 \\
$k_{i}$ & $1 \mathrm{~min}^{-1}$ & $q$ & $4 \times 10^{3}$ \\
$k_{t}$ & $0.9 \mathrm{~min}^{-1}$ & $h$ & 5 \\
$k_{e}$ & $4 \mathrm{~min}^{-1}$ & $\alpha$ & 3 \\
$D$ & $4 \times 10^{-6} \mathrm{~cm}^{2} / \mathrm{sec}$ & $\lambda$ & 0.01 \\
& & $\theta$ & 0.01 \\
& & $\epsilon$ & 0.15 \\
\hline
\end{tabular}

Remark 2..1. Let us remark that in the quasi-steady condition:

$$
w=\frac{k_{e} h}{k_{t}} u
$$


the system (2.6)-(2.8) reduces, in the homogeneous spatially case, to two equations for the variables $r$ and $u$. For the parameter values as in Table 1, the dynamics of the variable u is much faster than the one of $r$. In a straightforward manner one can check that the nullclines of the reduced system give rise to a monostable excitable medium. The unique equilibrium point is placed to the leftmost branch of the curve $\frac{d u}{d t}=0$, but close to its local maximum (see Figure 1).

An interesting feature of this reduced system is the small value for the threshold of excitability.
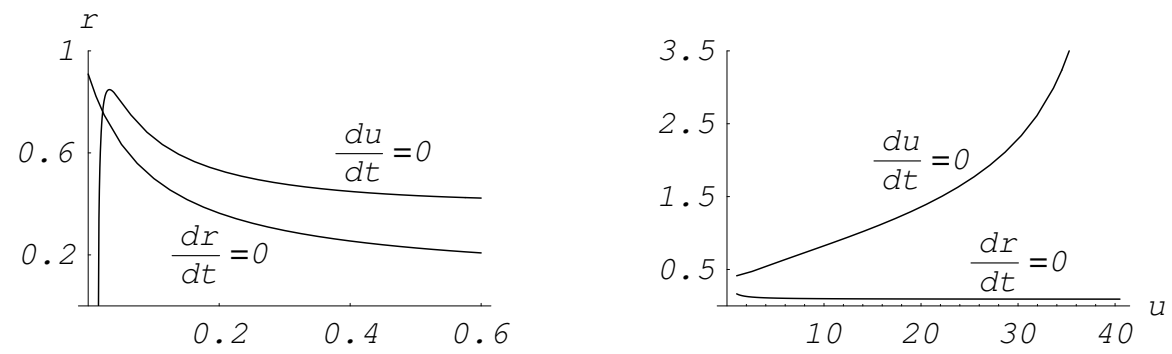

Figure 1: The nullclines corresponding to the system (2.6)-(2.8) in the homogeneous spatially case and the quasi-steady condition (2.9). The parameter values are the same as in Table 1.

\subsection{Mathematical analysis. Existence of pulse-like solutions.}

In this Subsection we present briefly the results concerning the existence of pulse-like solution for the system (2.6)-(2.8) (for more details see [31]).

We introduce a new time scale $\tau$ and dimensionless space variable $\widetilde{x}$ by means of:

$$
\tau=\left(k_{i}+k_{t}\right) \cdot t, \quad \widetilde{x}=\sqrt{\frac{k_{i}+k_{t}}{D}} \cdot y
$$

In the model (2.6)-(2.8) there are many parameters of different sizes and a priori is not clear which combination of these parameters can be assumed to be small or large. For this reason, we rewrote it in a way in which the new dependent variables are of order one. Then, rescaling the variables $r, w, u$ in the following way:

$$
\begin{aligned}
r(y, t) & =\frac{L_{2}(m+1)}{\left(1+L_{2}\right)(m+d)} \cdot R(\widetilde{x}, \tau) \\
w(y, t) & =\frac{M k_{e} h}{k_{t}} \cdot W(\widetilde{x}, \tau) \\
u(y, t) & =M \cdot U(\widetilde{x}, \tau)
\end{aligned}
$$

and taking into account the formulae (2.4), (2.5), the equations (2.6)-(2.8) become, after rearranging some terms: 


$$
\begin{aligned}
\frac{\partial R}{\partial \tau} & =\kappa(U+\mathcal{P} \varepsilon)\left[\frac{\mu(U+\varepsilon)-(U+d \varepsilon) R}{\left(U+\frac{\varepsilon}{c}\right)(U+\varepsilon)}\right] \\
\frac{\partial W}{\partial \tau} & =\frac{b \varepsilon(U+\varepsilon)^{2}+\Theta R^{2} U^{2}}{(U+\varepsilon)^{2}+\Lambda R^{2} U^{2}}-W \\
\frac{\partial U}{\partial \tau} & =\frac{\partial^{2} U}{\partial \widetilde{x}^{2}}+\Gamma[W-U]
\end{aligned}
$$

where $M$ is the largest root of the equation

$$
(u-b)(u+1)^{2}-\left((m+1)^{2} u^{2}(B-u)\right) /(m+d)^{2}=0,
$$

$m$ is the unique positive root of the equation $A(x-b)(x+d)^{2}-x^{2}(B-x)=0$ and the other parameter definitions are given in Table 2.

Our goal was to define a singular perturbation problem for the system of equations (2.11)(2.13) consistent with the values of the parameters in Table 1 . The system parameters must satisfy some rather stringent inequalities in order to give rise to a monotone excitable medium, namely:

$$
\begin{gathered}
1 \simeq 4 b B<A(2 b+d)^{2}<4 b B+4 b B\left(\frac{b+d}{2 b+d}\right)^{2} \\
3 b \ll 2 d-b<\frac{B}{A}<2 d-b+\sqrt{3 d(d-2 b)}<1
\end{gathered}
$$

These relations between the parameters of the system (2.11)-(2.13) are essential for obtaining the type of pulse-like dynamics. Reversing some of them, the nullclines structure can be changed in and this would have as a consequence that the system ceases to be excitable, or that the stability of the steady state is lost due to the onset of a bifurcation. 
Table 2. Orders of magnitudes and parameters numerical values.

\begin{tabular}{|c|c|c|c|c|}
\hline Parameter & Definition & $\begin{array}{l}\text { Parameters } \\
\text { relations }\end{array}$ & $\begin{array}{l}\text { Magnitude } \\
\text { order }\end{array}$ & $\begin{array}{l}\text { Numerical } \\
\text { value } \\
\text { (from Table 1) }\end{array}$ \\
\hline \multicolumn{5}{|c|}{ Small parameters } \\
\hline$\kappa$ & $k_{2}\left(1+L_{2}\right) /\left(k_{i}+k_{t}\right)$ & & $\ll 1$ & 0.0023 \\
\hline$\mu$ & $(m+d) /(m+1)$ & $\mu \sim d$ & $\ll 1$ & 0.1274 \\
\hline$\varepsilon$ & $M^{-1}$ & $\varepsilon \simeq \mu$ & $\ll 1$ & 0.1258 \\
\hline$d$ & $\left(1+L_{1}\right) /\left(c+L_{1}\right)$ & $d \gg 1 / c$ & $\ll 1$ & 0.1 \\
\hline$b$ & $\left(\alpha q \sigma k_{t} \lambda \theta\right) /\left(k_{e} h\left(k_{i}+k_{t}\right)(1+\alpha \theta)\right)$ & $8 b \simeq \varepsilon$ & $\ll 1$ & 0.0157 \\
\hline \multicolumn{5}{|c|}{ Parameters of order one } \\
\hline$\Gamma$ & $\overline{k_{e} /\left(k_{i}+k_{t}\right)}$ & & $\simeq 1$ & 2.1052 \\
\hline$\Lambda$ & $\left(A \mu^{2}\right)^{-1}$ & & $\simeq 1$ & 0.2966 \\
\hline$\Theta$ & $\Lambda B \varepsilon$ & & $\simeq 1$ & 1.5087 \\
\hline$\kappa \mathcal{P}$ & $k_{1}\left(1+L_{2}\right) /\left(k_{i}+k_{t}\right)$ & & $\simeq 1$ & 0.2315 \\
\hline \multicolumn{5}{|c|}{ Parameters much larger than one } \\
\hline $\mathcal{P} \varepsilon$ & $k_{1} /\left(M k_{2}\right)$ & & $\gg 1($ or $\simeq 1)$ & 12.582 \\
\hline $\mathcal{P}$ & $k_{1} / k_{2}$ & & $\gg 1$ & 100 \\
\hline$A$ & $(1+\alpha \theta)\left(1+L_{2}\right)^{2} /\left(\epsilon L_{2}^{2}(1+\alpha)\right)$ & & $\gg 1$ & 207.7 \\
\hline$B$ & $\left(\alpha q \sigma k_{t}\right) /\left(k_{e} h\left(k_{i}+k_{t}\right)(1+\alpha)\right)$ & & $\gg 1$ & 40.5 \\
\hline$c$ & & & $\gg 1$ & 100 \\
\hline
\end{tabular}

Let us now look for pulse-like solutions of the system (2.11)-(2.13) under the assumptions for the parameter magnitude orders as in Table 2. If a pulse solution exists, it can be written in the form

$$
R(\widetilde{x}, \tau)=R(x), \quad W(\widetilde{x}, \tau)=W(x), \quad U(\widetilde{x}, \tau)=U(x)
$$

where $x=\widetilde{x}-\mathcal{V} \tau$ and the pulse speed $\mathcal{V}>0$ is to be determined in the course of the analysis $(\mathcal{V}$ depends on the parameters of the system).

Substituting the pulse-form (2.16) into (2.11)-(2.13), the system becomes:

$$
\begin{aligned}
& -\mathcal{V} R^{\prime}=\kappa(U+\mathcal{P} \varepsilon)\left[\frac{\mu(U+\varepsilon)-(U+d \varepsilon) R}{\left(U+\frac{\varepsilon}{c}\right)(U+\varepsilon)}\right] \\
& -\mathcal{V} W^{\prime}=\frac{b \varepsilon(U+\varepsilon)^{2}+\Theta R^{2} U^{2}}{(U+\varepsilon)^{2}+\Lambda R^{2} U^{2}}-W \\
& -\mathcal{V} U^{\prime}=U^{\prime \prime}+\Gamma[W-U]
\end{aligned}
$$

where primes denote differentiation with respect to $x$. This is an eigenvalue problem to determine the value (or values) of $\mathcal{V}$ such that a non-negative solution $(R, W, U)$ of (2.17)-(2.19) exists and satisfies: 


$$
\lim _{|x| \rightarrow \infty} R(x)=R_{s}, \quad \lim _{|x| \rightarrow \infty} W(x)=W_{s}, \quad \lim _{|x| \rightarrow \infty} U(x)=U_{s}
$$

where $\left(R_{s}, W_{s}, U_{s}\right) \simeq(1, b \varepsilon, b \varepsilon)$ are the values for the unique spatially homogeneous equilibrium state of (2.11)-(2.13).

A schematic diagram of the $U$ pulse-like solution of (2.17)-(2.19) is shown in Figure 2, where the relevant regions are labeled.

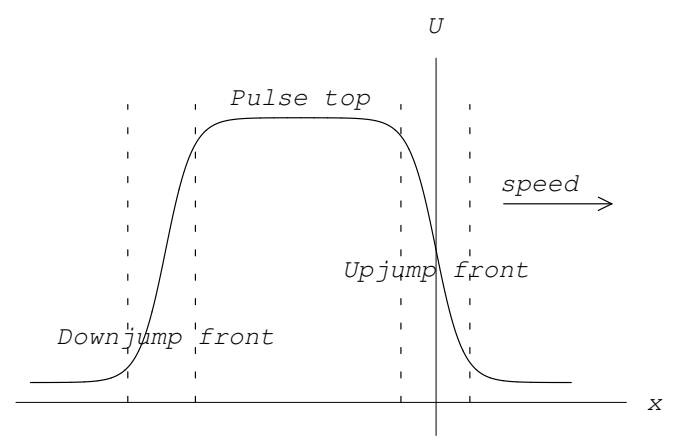

Figure 2: Schematic diagram of a pulse-like solution.

\subsubsection{Upjump front (fast transition)}

This thin region of the pulse, of size $\varepsilon^{\frac{1}{4}}$, is characterized by the abrupt change of the variables $W, U$ of an amount of order one, while the variable $R$ remains approximately constant, $R=R_{s}$. Hence, in this region the system (2.18)-(2.19) reduces to

$$
\begin{aligned}
& -\mathcal{V} W^{\prime}(x)=\frac{b \varepsilon(U+\varepsilon)^{2}+\Theta R_{s}^{2} U^{2}}{(U+\varepsilon)^{2}+\Lambda R_{s}^{2} U^{2}}-W \\
& -\mathcal{V} U^{\prime}(x)=U^{\prime \prime}+\Gamma[W-U]
\end{aligned}
$$

We seek a solution of the system (2.21)-(2.22) such that

$$
\begin{aligned}
& \lim _{x \rightarrow-\infty} U(x)=a, \quad \lim _{x \rightarrow-\infty} W(x)=a \\
& \lim _{x \rightarrow+\infty} U(x)=b \varepsilon, \quad \lim _{x \rightarrow+\infty} W(x)=b \varepsilon
\end{aligned}
$$

where

$$
a=\frac{\Theta R_{s}^{2}}{1+\Lambda R_{s}^{2}} \simeq 1
$$


Region I ( $U \gg \varepsilon$ ) Letting $\varepsilon \rightarrow 0$, the system (2.21)-(2.22) can be approximated in this first sublayer by

$$
\begin{aligned}
-\mathcal{V} W_{x} & =a-W \\
-\mathcal{V} U_{x} & =U_{x x}+\Gamma[W-U]
\end{aligned}
$$

where $a$ is given in (2.25) and we rewrite the limit conditions (2.23)-(2.24) as

$$
\begin{array}{ll}
\lim _{x \rightarrow-\infty} U(x)=a, & \lim _{x \rightarrow-\infty} W(x)=a \\
\lim _{x \rightarrow+\infty} U(x)=0, & \lim _{x \rightarrow+\infty} W(x)=0
\end{array}
$$

The problem (2.26)-(2.27) is linear and it can be explicitly solved. It has the solution:

$$
\begin{aligned}
& W(x)=a-C e^{\frac{x}{\mathcal{V}}} \\
& U(x)=a+C \frac{\Gamma \mathcal{V}^{2}}{1+\mathcal{V}^{2}(1-\Gamma)} e^{\frac{x}{\mathcal{V}}}+K e^{\frac{1}{2}\left(-\mathcal{V}+\sqrt{\mathcal{V}^{2}+4 \Gamma}\right) x}
\end{aligned}
$$

where

$$
\begin{aligned}
C & =\frac{2 a\left[1+\mathcal{V}^{2}(1-\Gamma)\right]}{\mathcal{V}\left[\sqrt{\mathcal{V}^{2}+4 \Gamma}-(2 \Gamma-1) \mathcal{V}\right]} \geqslant 0 \\
K & =\frac{a\left(\sqrt{\mathcal{V}^{2}+4 \Gamma}+\mathcal{V}\right)}{(2 \Gamma-1) \mathcal{V}-\sqrt{\mathcal{V}^{2}+4 \Gamma}}
\end{aligned}
$$

The solutions (2.30) and (2.31) satisfy also the limit condition (2.28), but they do not satisfy the boundary conditions (2.29) because, for $x \rightarrow+\infty$ the variable $U$ becomes of order $\varepsilon$ or smaller and the system (2.26)-(2.27) cease to be valid. For this region we shall introduce another suitable boundary sublayer.

Near to the point $x=0$, the variable $U$ behaves quadratically on $x$ due to (2.31), and $W$ is a linear function of $x$ (see Figure 3). This fact is relevant for matching with the next sublayer $(U \simeq \varepsilon)$.

Region II $(U \simeq \varepsilon)$ : a key non-linear eigenvalue problem This region is the important one for determining the upjump pulse speed. The following change of variables

$$
\mathcal{V}=\varepsilon^{-\frac{1}{4}} \cdot v, \quad x=\varepsilon^{\frac{1}{4}} v^{-1} \cdot z, \quad U(x)=\varepsilon \cdot u(z), \quad W(x)=\varepsilon^{\frac{1}{2}} \frac{\Theta R_{s}^{2}}{v^{2}} \cdot w(z)
$$




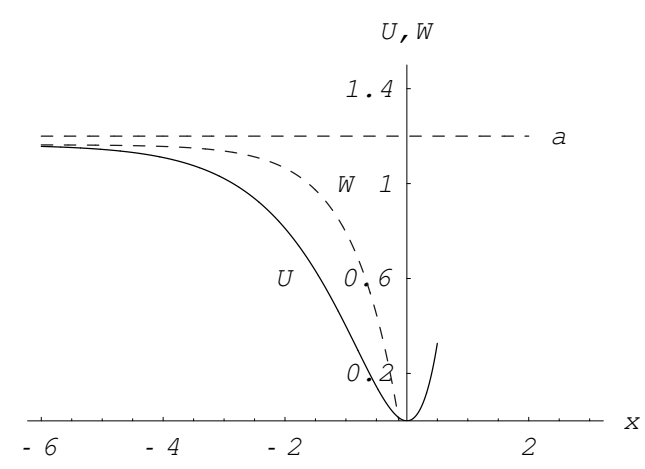

Figure 3: The shape of the solutions $W$ and $U$ given by (2.30)-(2.31) (the curves are obtained for the parameter values as in Table 1).

transforms the system (2.17)-(2.19), together with the matching conditions with the previous sublayer (in the intermediate limit $x \rightarrow 0, \eta \rightarrow-\infty, \varepsilon \rightarrow 0$ ) and the boundary conditions, into the following non-linear eigenvalue problem:

$$
\begin{aligned}
& -w_{z}=\frac{u^{2}}{(u+1)^{2}+\varkappa u^{2}} \quad-\infty<z<\infty \\
& -u_{z}=u_{z z}+\chi^{-4} w \quad-\infty<z<\infty \\
& w(z) \sim-\frac{1}{1+\varkappa} z, \quad u(z) \sim \frac{\chi^{-4}}{2(1+\varkappa)} z^{2}, \quad z \rightarrow-\infty \\
& \lim _{z \rightarrow \infty} w(z)=0, \quad \lim _{z \rightarrow \infty} u(z)=0
\end{aligned}
$$

where

$$
\varkappa=\Lambda R_{s}^{2}, \quad \chi^{-4}=\frac{\Gamma \Theta R_{s}^{2}}{v^{4}}
$$

Theorem 2..2. For each $\varkappa \geqslant 0$ there exists at least a value of $\chi=\chi(\varkappa)>0$ such that (2.32)-(2.35) has a solution.

Proof. We sketch out some ideas for the proof (for details see [31]).

We use a standard "shooting" method. For each fixed $\varkappa$ and $\chi$, there exists a unique positive solution of the system (2.32)-(2.33) satisfying the limit condition (2.35). Such a solution has the following asymptotic behaviour

$$
\begin{aligned}
u(z) & \sim e^{-z}, \quad w(z) \sim \frac{1}{2} e^{-2 z}, \quad z \rightarrow+\infty \\
u_{z}(z) & \sim L(\chi) e^{-z}, \quad z \rightarrow-\infty
\end{aligned}
$$

where 


$$
L(\chi)=\chi^{-4} \int_{-\infty}^{\infty} w(\tau) e^{\tau} d \tau-1
$$

The integral in (2.38) converges and $L(\chi)$ is a continuous function of $\chi$.

We show that the function $L(\chi)$ changes the sign as $\chi$ varies from 0 to $+\infty$. In the limit $\chi \rightarrow 0^{+}$, the function $u$ becomes negative at some finite value of $z$ and when $\chi \rightarrow+\infty$, the function $u$ increases exponentially as $z$ decreases to $-\infty$. We illustrate this fact in Figure 4.

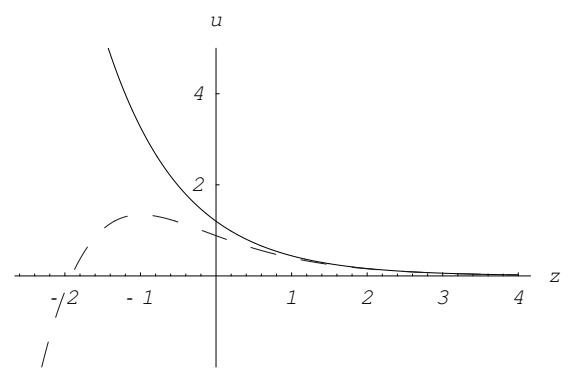

Figure 4: The curves represent the numeric $u$-approximation in the case $\chi \rightarrow+\infty$ (solid line) and respectively when $\chi \rightarrow 0^{+}$(dashed line) using the parameters from Table 1.

As $L(\chi)$ is a continuous function and changes the sign as $\chi$ varies from 0 to $+\infty$, there exists $\chi_{0}>0$ such that $L\left(\chi_{0}\right)=0$. For concluding the proof of the theorem, we show that for this value of $\chi, \chi=\chi_{0}$, we obtain the asymptotics

$$
\begin{aligned}
& w(z) \sim-\frac{1}{1+\varkappa} z, \quad \text { as } z \rightarrow-\infty \\
& u(z) \sim \frac{\chi^{-4}}{2(1+\varkappa)} z^{2}, \text { as } z \rightarrow-\infty
\end{aligned}
$$

This means that the matching with the previous sublayer is possible.

Remark 2..3. Numerical computation strongly suggests that the function $L(\chi)$ is strictly decreasing and there exists a unique value of $\chi, \chi=\chi_{0}$, such that $L\left(\chi_{0}\right)=0$.

The shape of the variable $u$ is illustrated in Figure 5.

\subsubsection{Pulse top (high concentration of $W$ and $U$ )}

After the upjump, the variables $W, U$ remain close to their equilibrium values in the equations (2.18)-(2.19), for each value of $R$. As the variable $R$ becomes of order one at distances $x$ of order $\kappa^{-1} \varepsilon^{-1 / 4}$, we introduce a new rescaling as follows

$$
\hat{x}=\frac{\kappa \varepsilon^{1 / 4}}{v} \cdot x
$$




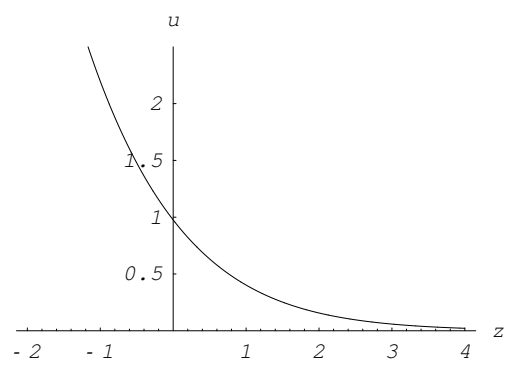

Figure 5: The shape of the solution $u$ of the system (2.32)-(2.35) (the curve is obtained by numerical computation for the parameter values as in Table 1).

Taking into account Table 2, the system (2.17)-(2.19) reduces in the considered asymptotic limit to

$$
\begin{aligned}
R^{\prime}(\hat{x}) & =\frac{R}{U} \cdot(U+\mathcal{P} \varepsilon) \\
W(\hat{x}) & =U(\hat{x})=\frac{\Theta R^{2}}{1+\Lambda R^{2}}
\end{aligned}
$$

where primes denote differentiation with respect to $\hat{x}$. The matching with the upjump requires

$$
\begin{aligned}
R(0) & =R_{s} \\
\lim _{\hat{x} \rightarrow 0} U(\hat{x}) & =\lim _{x \rightarrow-\infty} U(x)=a \\
\lim _{\hat{x} \rightarrow 0} W(\hat{x}) & =\lim _{x \rightarrow-\infty} W(x)=a
\end{aligned}
$$

The solution of (2.39)-(2.40), that satisfies the condition (2.41), is given by

$$
\begin{gathered}
R(\hat{x})=\sqrt{\frac{\mathcal{P} \varepsilon}{\Theta+\Lambda \mathcal{P} \varepsilon}\left[e^{\frac{2(\Theta+\Lambda \mathcal{P} \varepsilon)}{\Theta}\left(\hat{x}-\hat{x}_{1}\right)}-1\right]} \\
U(\hat{x})=W(\hat{x})=\frac{\Theta}{\Lambda} \cdot \frac{e^{\frac{2(\Theta+\Lambda \mathcal{P} \varepsilon)}{\Theta}\left(\hat{x}-\hat{x}_{1}\right)}-1}{e^{\frac{2(\Theta+\Lambda \mathcal{P} \varepsilon)}{\Theta}\left(\hat{x}-\hat{x}_{1}\right)}+\frac{\Theta}{\Lambda \mathcal{P} \varepsilon}}
\end{gathered}
$$

where

$$
\hat{x}_{1}=\frac{\Theta}{2(\Theta+\Lambda \mathcal{P} \varepsilon)} \ln \frac{\mathcal{P} \varepsilon}{\mathcal{P} \varepsilon+R_{s}^{2}(\Theta+\Lambda \mathcal{P} \varepsilon)}<0
$$

The approximation formulae (2.44) and (2.45) are valid in the interval $\hat{x}_{1} \leqslant \hat{x} \leqslant 0$. The shape of the solution $U$ in this region is given in Figure 6. 


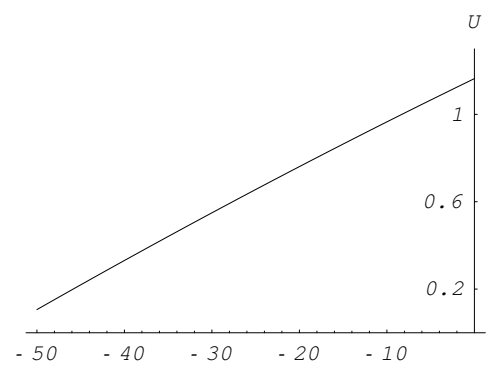

Figure 6: The shape of the solution $U(\hat{x})$ of the system (2.32)-(2.35) (the curve is obtained by numerical computation for the parameter values as in Table 1).

\subsubsection{Downjump front}

In the last part of the pulse the variables $W, U$ do not change adiabatically with $R$ any longer, and all the variables return to the equilibrium state $\left(R_{s}, W_{s}, U_{s}\right)$. The width of this layer is of order $\varepsilon^{-1 / 4}$ up to logarithmic corrections. Due to the complexity of the nonlinearities in (2.17)-(2.19), the detailed study of this region requires to decompose it in several sublayers taking into account the orders of magnitude of the variables $(U \simeq \varepsilon, U R \simeq \mu \varepsilon, U \simeq d \varepsilon, b \varepsilon \ll U \ll d \varepsilon, U \sim b \varepsilon$ ).

This analysis is quite cumbersome and we shall not present it in detail here. The main reason is that the description of the downjump front is independent on the value of $v$, up to a rescaling of the lenght, so the effect of this boundary layer for the shape of the pulse is very small.

Only by self-consistency of the pulse construction, we indicate in Figure 7 the behaviour of the solution $U$ in the first sublayer of this region.

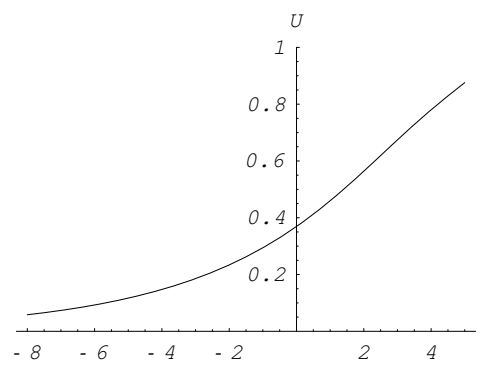

Figure 7: The shape of the solution $U$ in the first sublayer of the downjump front (the curve is obtained by numerical computation for the parameter values as in Table 1).

\subsubsection{Pulse speed. Comparison with experimental data.}

The formulae for the pulse speed and pulse width, as functions of the biochemical parameters of the initial problem, are given by 


$$
\begin{aligned}
\text { speed }_{\text {cAMP pulse }} & =\sqrt{D} \cdot\left(\frac{\alpha q \sigma k_{t}}{h(1+\alpha)}\right)^{1 / 4} \cdot \varkappa^{1 / 4} \cdot \chi(\varkappa) \\
\text { width }_{\text {cAMP pulse }} & =\frac{\sqrt{D}}{2 k_{2}\left(1+L_{2}\right)} \cdot\left(\frac{\alpha q \sigma k_{t}}{h(1+\alpha)}\right)^{1 / 4} \cdot\left(1-\frac{1}{\Upsilon}\right) \cdot \varkappa^{1 / 4} \cdot \chi(\varkappa) \cdot \ln (1+\Upsilon \varkappa)
\end{aligned}
$$

where

$$
\begin{aligned}
& \varkappa=\frac{(1+\alpha) \epsilon}{(1+\alpha \theta)} \cdot\left(\frac{L_{2}\left(c+L_{1}\right)}{1+L_{2}}\right)^{2} \cdot\left(\frac{2 \alpha q \sigma k_{t} \lambda \theta+k_{e} h\left(k_{i}+k_{t}\right)(1+\alpha \theta)}{2 \alpha q \sigma k_{t} \lambda \theta\left(c+L_{1}\right)+k_{e} h\left(k_{i}+k_{t}\right)(1+\alpha \theta)\left(1+L_{1}\right)}\right)^{2} \\
& \Upsilon=1+\frac{\alpha q \sigma k_{t} k_{2}}{k_{1} k_{e} h\left(k_{i}+k_{t}\right)(1+\alpha)}
\end{aligned}
$$

Taking into account the numerical values of the parameters given in Table 1 , we obtain $\varkappa \approx$ 0.2966 and the corresponding eigenvalue $\chi(\varkappa)$ of the problem (2.32)-(2.35), numerically computed, is $\chi \approx 0.3377$. Then, the numerical value for the pulse speed and width are

$$
\begin{aligned}
\text { speed }_{c A M P \text { pulse }} & \approx 162 \mu \mathrm{m} / \mathrm{min} \\
\text { width }_{\text {cAMP pulse }} & \approx 1848.5 \mu \mathrm{m}
\end{aligned}
$$

The experimental value for the $c A M P$ pulses speed is $250-300 \mu \mathrm{m} / \mathrm{min}$ (see [1], [2], [20], [30], [37]) and for the pulse width is about $1400 \mu \mathrm{m}$ (see [20]).

On can see that the the pulse speed and pulse width values obtained here are reasonable approximations and agree with the order of magnitude obtained in the experimental observations.

\section{A mathematical model for cAMP signalling in mound stage}

\subsection{Description of the model}

In [47], Vasiev and Weijer explain the cell movement pattern in the mound assuming that the cells move chemotactically in response to a scroll-shaped $c A M P$ wave rotating in the mound. To model propagating $c A M P$ waves, they consider this FitzHugh-Nagumo type system:

$$
\begin{aligned}
& \frac{\partial g}{\partial t}=D \Delta g-k_{g}\left(g-g_{0}\right)\left(g-g_{1}\right)\left(g-g_{2}\right)-k_{r} r \\
& \frac{\partial r}{\partial t}=\left(g-g_{0}-r\right) / \tau
\end{aligned}
$$

Here the variable $g(y, t)$ is assumed to define the level of extracellular $c A M P$, and the variable $r(y, t)$ represents either the proportion of active and inactive $c A M P$ receptors (like in [33]), or 
activated $\alpha$ subunits of the inhibitory G-proteins (like in [39], [42]). The parameters appearing in (3.1)-(3.2) have the following meanings: $D$ is the $c A M P$ diffusion coefficient, $k_{g}$ and $k_{r}$ define the rate of $c A M P$ production and hydrolysis respectively, $\tau$ is a time scaling factor for the variables $g$ and $r$. The dimensionless parameters considered in [47] are: $D=1, g_{0}=0.3, g_{1}=0.35$, $g_{2}=1.3, k_{r}=1.5, \tau=4$ and $k_{g}$ take values between 5.4 and 6.0. They are chosen to fit with experimentally measurable data.

Making the following transformations

$$
x=\sqrt{\frac{k_{g}}{D}} \cdot y, \quad s=k_{g} \cdot t, \quad u(x, s)=g(y, t)-g_{0}, \quad w(x, s)=\frac{k_{r}}{k_{g}} \cdot r(y, t)
$$

from (3.1)-(3.2) we obtain the following system:

$$
\begin{aligned}
\frac{\partial u}{\partial s} & =\Delta u+u(u-\varepsilon)(a-u)-w \\
\frac{\partial w}{\partial s} & =\varepsilon^{1+\delta}(u-u w)
\end{aligned}
$$

where the parameter definitions and the numerical values are given in Table 3.

\begin{tabular}{|l|r|}
\hline \multicolumn{2}{|c|}{ Table 3. Numerical parameter values. } \\
\hline $\begin{array}{l}\text { Dimensionless } \\
\text { parameter }\end{array}$ & \multicolumn{1}{|c|}{$\begin{array}{l}\text { Numerical } \\
\text { value }\end{array}$} \\
\hline$\varepsilon=g_{1}-g_{0}$ & 0.05 \\
$\varepsilon^{1+\delta}=k_{r} / \tau k_{g}^{2}$ & $0.013-0.01$ \\
$a=g_{2}-g_{0}$ & 1 \\
$u=k_{g} / k_{r}$ & $3.6-4$ \\
\hline
\end{tabular}

The parameters $\varepsilon$ and $\varepsilon^{1+\delta}$ should be considered like independent parameters. We use in a abusive manner these notations because we want to suggest that $\varepsilon^{1+\delta}$ is much smaller that $\varepsilon$. The idea in the following analysis is that we have the following asymptotic relations $0<\varepsilon^{1+\delta} \ll \varepsilon \ll$ 1.

\subsection{Mathematical analysis}

We consider the system (3.3)-(3.4) with $a=1$, in one spatial dimension:

$$
\begin{aligned}
\frac{\partial u}{\partial s} & =\Delta g+u(u-\varepsilon)(1-u)-w \\
\frac{\partial w}{\partial s} & =\varepsilon^{1+\delta}(u-u w)
\end{aligned}
$$


Remark 3..1. The system (3.5)-(3.6) is the FitzHugh-Nagumo system with a small threshold of excitability. There is an exhaustive literature about this widely known model describing an excitable medium (see, for example, [4], [5], [6], [21], [23], [24], [35], [48]). It is known that the pulselike solutions consist in two thin layers (upjump and downjump) where the fast variable changes abruptly from the excitable to the excited state and viceversa respectively. The essential fact is that the wave speed of the upjump must be the same as of the downjump. This abrupt transitions are separated by a region where the fast variable remains attached to its equilibrium value and the slow variable has a change of order one.

Considering the travelling wave coordinate $\xi=x-\mathcal{V} s$, the system (3.5)-(3.6) becomes

$$
\begin{array}{r}
u^{\prime \prime}+\mathcal{V} u^{\prime}+u(u-\varepsilon)(1-u)-w=0 \\
\mathcal{V} w^{\prime}+\varepsilon^{1+\delta}(u-u w)=0
\end{array}
$$

where the primes denote differentiation with respect to $\xi$.

Here we are not interested in obtaining a complete description of a pulse solution for (3.7)-(3.8) in the limit $\varepsilon \rightarrow 0$, we only want to determine the pulse speed, so we look only at the pulse upjump.

If we put $\varepsilon^{1+\delta}=0$ in (3.8) we obtain $w^{\prime}(\xi)=0$. We assume that $\lim _{\xi \rightarrow \infty} w(\xi)=0$, hence $w$ is everywhere zero. From (3.7)-(3.8) we get

$$
u^{\prime \prime}+\mathcal{V} u^{\prime}+u(u-\varepsilon)(1-u)=0
$$

This is the bistable equations with the value of the threshold equal to $\varepsilon$. Since the wave solutions are invariant up to translations, let us take $u(0)=1 / 2$ and it is known that this equation admits one unique wavefront solution satisfying the limit conditions

$$
\lim _{\xi \rightarrow \infty} u(\xi)=0, \quad \lim _{\xi \rightarrow-\infty} u(\xi)=1
$$

which it is given by the formula

$$
u(\xi)=\frac{1}{1+e^{\mathcal{V}_{0} \xi}}
$$

with the wave speed

$$
\mathcal{V}_{0}=\frac{1}{\sqrt{2}}(1-2 \varepsilon)
$$

(see, for example [16], [35]).

Although the speed and wavefront solution formulae are well known, let us make some remarks about the equation (3.9). Starting with this equation and considering $\varepsilon$ as a small parameter, one can work out a procedure based on perturbations methods in order to obtain the travelling wave solution (3.11), and the formula for the wave speed (3.12). More precisely, if we assume that $u$ and $\mathcal{V}$ are perturbation series in $\varepsilon$, then they have expansions of the form 


$$
\begin{aligned}
u(\xi ; \varepsilon) & =u_{0}(\xi)+\varepsilon u_{1}(\xi)+\varepsilon^{2} u_{2}(\xi)+\ldots \\
\mathcal{V}(\varepsilon) & =\mathcal{V}_{0}+\varepsilon \mathcal{V}_{1}+\varepsilon_{2}^{2} \mathcal{V}_{2}+\ldots
\end{aligned}
$$

Considering the case when $\mathcal{V}$ is strictly of order unity, this means that $\mathcal{V}_{0}>0$. The boundary conditions at $\pm \infty$, the fact that $u(0)=1 / 2$ and (3.13)-(3.14) give the conditions for the $u_{i}(x)$, $i=0,1,2, \ldots$ :

$$
\begin{array}{llc}
\lim _{\xi \rightarrow \infty} u_{0}(\xi)=0, & \lim _{\xi \rightarrow-\infty} u_{0}(\xi)=1, & u_{0}(0)=\frac{1}{2} \\
\lim _{\xi \rightarrow \infty} u_{i}(\xi)=0, & \lim _{\xi \rightarrow-\infty} u_{i}(\xi)=0, & \text { for } i=1,2, \ldots \\
u_{0}(0)=\frac{1}{2}, & u_{i}(0)=0, & \text { for } i=1,2, \ldots
\end{array}
$$

By substituting (3.13), (3.14) into (3.9) and equating powers of $\varepsilon$, we get the following eigenvalue problems

$$
\begin{aligned}
O(1): & u_{0}^{\prime \prime}+\mathcal{V}_{0} u_{0}^{\prime}+u_{0}^{2}\left(1-u_{0}\right)=0 \\
O(\varepsilon): & u_{1}^{\prime \prime}+\mathcal{V}_{0} u_{1}^{\prime}+u_{0}\left(2-3 u_{0}\right) u_{1}=u_{0}\left(1-u_{0}\right)-\mathcal{V}_{1} u_{0}^{\prime} \\
O\left(\varepsilon^{2}\right): & u_{2}^{\prime \prime}+\mathcal{V}_{0} u_{2}^{\prime}+u_{0}\left(2-3 u_{0}\right) u_{2}=u_{1}^{2}\left(3 u_{0}-1\right)-u_{1}\left(2 u_{0}-1\right)-\mathcal{V}_{1} u_{1}^{\prime}-\mathcal{V}_{2} u_{0}^{\prime}
\end{aligned}
$$

and so on for higher orders of $\varepsilon$. Using the explicit solution formulae for the corresponding homogeneous equations, matching conditions and orthogonality restrictions, it can be shown that

$$
\begin{gathered}
u_{0}(\xi)=\frac{1}{1+e^{\mathcal{V}_{0} \xi}}, \quad u_{i}(\xi)=0, \quad \text { for } i=1,2, \ldots \\
\mathcal{V}_{0}=\frac{1}{\sqrt{2}}, \quad \mathcal{V}_{1}=-\sqrt{2}, \quad \mathcal{V}_{i}=0 \quad \text { for } i=1,2, \ldots
\end{gathered}
$$

and in this way the formulae (3.11) and (3.12) can be recover by using perturbation methods.

Remark 3..2. We mention that (3.18) is the Zeldovich equation and it is known that this equation admits precisely one distinct wavefront solution from 1 to 0 for every wave speed $\mathcal{V}_{0} \geqslant 1 / \sqrt{2}$ and no such solution for any wave speed $\mathcal{V}_{0}<1 / \sqrt{2}$ (see, for example [16], [35]).

The result can be formulated in a more general manner (see [16]):

Theorem 3..3. The equation (3.9) admits exactly one distinct wavefront solution from $\varepsilon$ to 0 for all wave speeds $\mathcal{V} \leqslant-2 \sqrt{\varepsilon(1-\varepsilon)}$, exactly one distinct wavefront solution from 1 to $\varepsilon$ for all wave speeds $\mathcal{V} \geqslant(1+\varepsilon) / \sqrt{2}$, exactly one distinct wavefront solution from 1 to 0 with wave speeds $\mathcal{V}=(1-2 \varepsilon) / \sqrt{2}$ and no other decreasing wavefront solutions. 


\subsection{Pulse speed. Comparison with experimental data.}

The formula for the pulse speed, as a function of the biochemical parameters of the initial problem, is given by

$$
\text { speed }_{\text {cAMP pulse }}=\left(1-2\left(g_{1}-g_{0}\right)\right) \sqrt{D k_{g} / 2}
$$

Taking into account the parameter numerical values given in [47], we obtain

$$
\text { speed }_{\text {cAMP pulse }} \approx 1.478-1.558
$$

The measured value of the $c A M P$ pulses speed in mould of $D d$ in [47] is 1.2 space units per time unit, which agrees with the order of magnitude obtained in (3.24).

\section{Conclusions}

The goal of this work was to find analytical formulae in terms of biological parameters for two 1-dimensional spatial models of $c A M P$ signalling. The common feature of the two models is the threshold that approaches to zero in the considered asymptotic limit. However, there are also some differences between them. The main one is that the presence of three equations in the kinetics of the first model, instead of two, makes harder to derive closed form formulae. In the case of the FHN system for obtaining a nondeformed pulse is essential that the speed of the upjump and downjump, where the fast variable changes abruptly, to be the same. On the contrary, in the first model considered in this paper, the downjump does not require any choice of the pulse speed, up to a rescaling of the length.

\section{Acknowledgements}

This work was partially supported by the RTN "Modeling, Mathematical Methods and Computer Simulation of Tumour Growth and Therapy" (MRTN-CT-2004-503661), DGES Grant MTM200405634 and CEEX Grant PC-D11-PT01-836.

\section{References}

[1] F. Alcantara, M. Monk. Signal propagation during aggregation in the slime mould Dictyostelium discoideum. J. Gen. Microbiol., 85 (1974), 321-334.

[2] E. Ben-Jacob, I. Cohen. Cooperative self-organization of microorganisms. Advances in Physics, 49 (2000), 395-554.

[3] J.T. Bonner. The cellular slime molds. Princeton University Press, Princeton, 1967. 
[4] R.G. Casten, H. Cohen,P.A. Lagerstrom. Perturbation analysis of an approximation to the Hodgkin-Huxley theory. Quart. Appl. Math., 32 (1975), 365-402.

[5] M. Courtemanche, W. Skaggs, A.T. Winfree. Stable three-dimensional action potential circulation in the Fitzhugh-Nagumo model. Physica D, 41 (1990), 173-182.

[6] E. Cytrynbaum, J.P. Keener. Stability conditions for the travelling pulse: Modifying the restitution hypothesis. Chaos, 12 (2002), 788-799.

[7] J.C. Dallon, H.G. Othmer. A discrete cell model with adaptive signalling for aggregation of Dictyostelium discoideum”. Phil. Trans. R. Soc. Lond. B, 352 (1997), 391-417.

[8] D. Dormann, J.-Y. Kim, P.N. Devreotes, C.J. Weijer. cAMP receptor affinity controls wave dynamics, geometry and morphogenesis in Dictyostelium. J. Cell Science, 114 (2001), 25132523.

[9] D. Dormann, C.J. Weijer. Propagating chemoattractant waves coordinate periodic cell movement in Dictyostelium slugs. Development, 128 (2001), 4535-4543.

[10] J.A. Durston. Pacemaker activity during aggregation in Dictyostelium discoideum. Developmental Biology, 37 (1974), 225-235.

[11] J.A. Durston. Pacemaker mutants of Dictyostelium discoideum. Developmental Biology, 38 (1974), 308-319.

[12] M. Dworkin, K.H. Keller. Solubility and diffusion coefficient of adenosine 3':5' monophosphate. J. Biol. Chem., 252 (1977), 864-865.

[13] M. Falcke, H. Levine. Pattern selection by gene expression in Dictyostelium discoideum. Phys. Rev. Lett., 80 (1998), 3875-3878.

[14] P. Fife. Mathematical Aspects of Reacying and Diffusing Systems, Springer Verlag, Berlin, 1979.

[15] G. Gerisch, D. Hülser, D. Malchow, U. Wick. Cell communication by periodic cyclic-AMP pulses. Phil. Trans. R. Soc. Lond. B, 272 (1975), 181-192.

[16] B.H. Gilding, R. Kersner. Travelling waves in nonlinear diffusion-convection reaction. Birkhäuser Verlag, Basel, 2004.

[17] A. Goldbeter, L.A. Segel. Unified mechanism for relay and oscillation of cyclic AMP in Dictyostelium discoideum. Proc. Natl. Acad. Sci. USA, 74 (1977), 1543-1547.

[18] P. Grindrod. Patterns and Waves. The theory and applications of reaction-diffusion equations. Clarendon Press, Oxford, 1991.

[19] P.S. Hagan, M.S. Cohen. Diffusion-induced morphogenesis in the development of Dictyostelium. J. Theor. Biol., 93 (1981), 881-908. 
[20] T. Höfer, J.A. Sherratt, P.K. Maini. Cellular pattern formation during Dictyostelium aggregation. Physica D, 85, (1995), 425-444.

[21] C.K.R.T. Jones. Stability of the travelling wave solution of the FitzHugh-Nagumo system. Trans. Amer. Math. Soc., 286 (1984), 431-469.

[22] J.P. Keener. Waves in excitable media. SIAM J. Appl. Math., 39 (1980), 528-548.

[23] J.P. Keener. A geometrical theory for spiral waves in excitable media. SIAM J. Appl. Math., 46 (1986), 1039-1056.

[24] J.P. Keener, J. Sneyd. Mathematical physiology. Springer-Verlag, New York, 1998.

[25] E.F. Keller, G.M. Odell. Necessary and sufficient conditions for chemotactic bands. Math. Biosci., 27 (1975), 309-317.

[26] E.F. Keller, G.M. Odell. Traveling bands of chemotactic bacteria revisited. J. Theor. Biol., 56 (1976), 243-247.

[27] E.F. Keller, L.A. Segel. Traveling bands of chemotactic bacteria: a theoretical analysis. J. Theor. Biol., 30 (1971), 235-248.

[28] K.J. Lee. Wave pattern selection in a excitable system. Phys. Rev. Lett., 79 (1997), 29072910.

[29] K.J. Lee, E.C. Cox, R.E. Goldstein. Competing patterns of signalling activity in Dictyostelium discoideum. Phys. Rev. Lett., 76 (1996), 1174-1177.

[30] H. Levine, L. Tsimring, D. Kessler. Computational modeling of mound development in Dyctiostelium. Physica D, 106 (1997), 375-388.

[31] G. Liţcanu, J.J.L. Velázquez. Singular perturbation analysis of cAMP signalling in Dictyostelium discoideum aggregates. J. Math. Biol., 52 (2006), 682-718.

[32] L. Ma, C. Janetopoulos, L. Yang, P.N. Devreotes, P.A. Iglesias. Two complementary, local excitation, global inhibition mechanisms acting in parallel can explain the chemoattractantinduced regulation of PI(3,4,5)P $P_{3}$ response in Dictyostelium cells. Biophys. J., 87 (2004), 3764-3774.

[33] J.L. Martiel, A. Goldbeter. A model based on receptor desensitization for cyclic AMP signalling in Dictyostelium cells. Biophys. J., 52 (1987), 807-828.

[34] P.B. Monk, H.G. Othmer. Cyclic AMP oscillations in suspension of Dictyostelium discoideum. Phil. Trans. R. Soc. Lond., 323 (1989), 185-224.

[35] J.D. Murray. Mathematical Biology, 3d edition, Springer-Verlag, Berlin, 2003. 
[36] S.C. Müller, T. Mair, O. Steinbock. Traveling waves in yeast extract and in cultures of Dictyostelium discoideum. Biophysical Chemistry, 72 (1998), 37-47.

[37] V. Nanjundiah. Cyclic AMP oscillations in Dictyostelium discoideum: models and observation. Biophysical Chemistry, 72 (1998), 1-8.

[38] H.G. Othmer, P. Schaap. Oscilatory cAMP signalling in the development of Dictyostelium discoideum. Comments on Theoretical Biology, 5 (1998), 175-282.

[39] H.G. Othmer, Y. Tang. A G-protein-based model for cAMP dynamics in Dictyostelium discoideum. Math. Biosciences, 120 (1994), 25-76.

[40] C.A. Parent, P.N. Devreotes. A cell's sense of direction. Science, 284 (1999), 765-770.

[41] J. Rinzel, D. Terman. Propagation phenomena in a bistable reaction-diffusion system. SIAM J. Appl. Math., 42 (1982), 1111-1137.

[42] Y. Tang, H.G. Othmer. Excitation, oscillations and wave propagation in a G-protein-based model of signal transduction in Dictyostelium discoideum. Phil. Trans. R. Soc. Lond. B, 349 (1995), 179-195.

[43] K.J. Tomchik, P.N. Devreotes. Adenosine 3',5'-monophosphate waves in Dictyostelium discoideum: a demonstration by isotope dilution-fluorography. Science, 212 (1981), 443-446.

[44] J.J. Tyson, K.A. Alexander, V.S. Manoranjan, J.D. Murray. Spiral waves of cyclic AMP in a model of slime mold aggregation. Physica D, 34 (1989), 193-207.

[45] J.J. Tyson, J.P. Keener. Singular perturbation theory of traveling waves in excitable media (a review). Physica D, 32 (1988), 327-361.

[46] J.J. Tyson, J.D. Murray. Cyclic-AMP waves during aggregation of Dictyostelium amoebae. Development, 106 (1989), 421-426.

[47] B. Vasiev, C.J. Weijer. Modeling chemotactic cell sorting during Dictyostelium discoideum mound formation. Biophys. J., 76 (1999), 595-605.

[48] A.I. Volpert, V.A. Volpert, V.A. Volpert. Traveling wave solutions of parabolic systems. Translations of Mathematical Monographs, AMS, Providence, 1994.

[49] A.T. Winfree. Wavefront geometry in excitable media. Physica D, 12 (1984), 321-332. 